$$
\text { DOE/CE/A15995-..... DOE/CE/15985-T3 }
$$
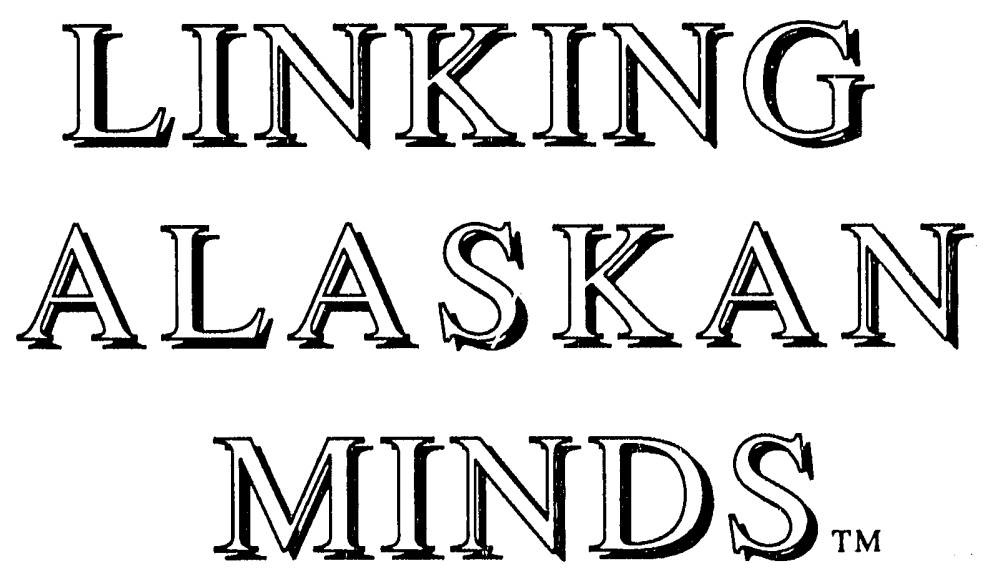

"How to Design and Establish a Computer Bulletin Board to Support Inventors."

A project supported by funding from the State's Initiatives Program

Department of Energy

Chicago Operations Office

9800 South Cass Avenue

Argonne, Illinois 60439

Prepared by the Alaska Inventors Association

P.O. Box

Anchorage, AK 9950 
This booklet is a "how-to"handbook to demonstrate the development of an interactive electronic bulletin board as a support network for independent inventors and small business inventors.

This will explain step-by-step, how Linking Alaskan MindsTM, the Alaskan model of an interactive computer bulletin board system, was developed and designed to serve as a successfully working, interactive computer bulletin board that links and supports independent inventors in Alaska.

\section{DISCLAIMER}

This report was prepared as an account of work sponsored by an agency of the United Siates Government. Neither the United States Gnvernment nor any agency thereof, nor any of their employees, makes any warranty, express or implied, or assumes any legal liability or responsibility for the accuracy, completeness, or usefulness of any information, apparatus, product, or process disclosed, or represents that its use would not infringe privately owned rights. Reference herein to any specific commercial product, process, or service by trade name, trademark, manufacturer, or otherwise does not necessarily constitute or imply its endorsement, recommendation, or favoring by the United States Government or any agency thereof. The views and opinions of authors expressed herein do not necessarily state or reflect those of the United States Government or any agency thereof. 


\section{Introduction to Linking Alaskan Minds-- $\underline{\text { An Overview }}$}




\section{Linking Alaskari Minds Project:}

The Alaska Inventors Association is attempting to develop an effective way to meet the divergent needs of inventors from across the state. Due to distance, variety of projects related to their individual communities economic make-up and the inability to network regularly, there is a tremendous demand for an innovative method for providing support and encouragement to inventors. An office in Anchorage will serve the major population base, however, there are numerous inventors in "hot spots" throughout Alaska who would not regularly benefit from that structure. We believe that an electronic "office" will link Alaskan minds providing a forum for encouragement and assistance to promote more invention and innovation and commercialization.

\section{Introducing Linking Alaskan Minds (The LAM)}

The The LAM Bulletin Board System (BBS) is a program that allows you to set up and run a remote access message system. Using your computer, a modem, and the Searchlight software, you'll have a complete bulletin board system that will

- Allow others to access your PC via telephone and exchange mail and other messages, even when you are not at your computer.

- Provide password access and multiple levels of security, enabling you to control exactly who has access to what information on your system.

- Allow unattended, error-free transfer of programs and data to and from your PC and other PC's via telephone.

-Provide a complete, user friendly environment that's easy enough for most people to use intuitively, without the need for any printed documentation or assistance from you. 


\section{The \\ Alaska Inventors Association}




\section{Mission Statement (Purpose)}

The mission of the Alaska Inventors Association is to serve as a catalyst, thereby enabling the identification and linkage of people to resources (technical, material, professional) necessary to facilitate the successful commercialization of Alaskan ideas.

\section{Philosophy}

Our philosophy stems from the recognition that successful Alaskan inventors and innovators have found it necessary to gain assistance, expertise and investment outside of Alaska. The founding board of AIA sought to counteract the transfer of technology out of state with the intention of creating an environment that would support an Alaska manufacturing distribution base. We believe Alaskans, through networking with other Alaskans can help find the resources to commercialize, in state, their innovations and inventions regardless of whether they originate in a garage or a sophisticated science lab. 


\section{Business Description:}

The Alaska Inventors Association is a non-profit, membership driven, statewide economic development organization which acts as a catalyst, creating an environment to enhance creative innovation and technology transfer for inventors of all ages throughout the state of Alaska. This catalyst will function by identifying and linking people and resources (technical, material, professional) necessary to facilitate successful commercialization of Alaskan ideas.

A premise basic to the Alaska Inventors Association is to not duplicate any existing services or assistance to inventors or innovators, rather it will support and bridge those services.

The Alaska Inventors Association pledges to work closely with those services that exist which are essential to the commercization of a product and make the highest and best use of each of those resources. 


\section{Organizational Goals and Objectives:}

\section{Serving as a Catalyst for Innovation/Invention}

- Raise the Profile of the Independent Inventor

- Stimulate Creativity/Problem Solving

- Promote Alaska as an "Idea Development Center" , fostering confidence in Alaskan know how and ability. MAKING IT HAPPEN IN ALASKA!

- Legislative Advocacy (Fraud, Intellectual Property Protection, Invention Evaluation Scams)

\section{Identifying Resources}

Identify the following:

- Ideas

- Inventors/Innovators

- Material

- Equipment

- Technical Expertise

- Facilities

- Funding

- Sources of Information

\section{Linking Resources}

- Resource Directory

- Linking Alaskan Minds (Network)

- Newsletter

- Chapters 


\section{Association History}

The AIA is a non-profit corporation, 501(c)3, established in 1988. The Association resulted from a need for a centralized forum for inventors and innovators to share resources and information essential to the success in the evaluation, financing and marketing of inventions. Although other states had well-established organizations that served these needs, Alaska had only two regional clubs that provided only limited networking and education opportunities. In January of 1988, the second in a series of seminars on patenting and marketing inventions was offered in Anchorage. Sponsored by the Loussac Library and the University of Alaska, Small Business Development Center, these seminars attracted over 65 inventors from the Anchorage area. The announcement of these seminars resulted in over 200 telephone inquiries requesting available information and future educational programs. Inventors expressed an interest in regular networking and educational forums directly related to innovation in their local communities and so the Alaska Inventors Association had begun.

In October of 1988, the first general membership meeting of the newly incorporated Alaska Inventors Association was held in Anchorage. The keynote speaker, Governor Steve Cowper addressed inquiries mad hy individual inventors. At that same time, the first monthly newletter of the Alaska Inventors Association was distributed to members. This meeting was audio conferenced to several locations throughout the state. The kick-off was a success. People had an opportunity to network and "good" information was disseminated about the patent process, protecting your idea and the marketability of inventions. 
At the same time that members were organizing the association, the state of Alaska was seeking diversified economic opportunities that would create and maintain jobs. State economic development specialists did not have to look too far to find developing inventions that varied in nature from being recreational to inventions on the cutting edge of technology in fields as diverse as bio-medical and energy efficiency. Alaskans had learned to "make-do" and were using these skills to develop new products and systems. Inventors and innovators could stimulate the economy through the development and marketing of their products and systems. 


\section{AKIA On-Line}

Translating services to an electronic bulletin board. 


\section{To Dial Up:}

Linking Alaskan Minds

(907) 694-6371

Protocol:

Dialing...

AT

OK

ATDT694-6371

CONNECT 1200

:::: 1200 Baud Connection ::::

Scarchlight BBS System Version 1.77A-M, 6-Dec-1990 Registration \# 1042

\section{Screen \#1}

Select Graphics Mode: [C]olor, [M]onochrome, or [N]one ? N

Enter your name, or type NEW

$>$ (YOUR NAME)

Password? ..... 


\section{Screen \#2}

\section{Welcome to Linking}

\section{Alaskan}

\section{Minds}

On the LAM BBS Welcomes (Your Name) on Mon, 4-29-91, 2:07 pm

You last called on 4-29-91

You are caller no. 420 , with $1 ? 0$ calls total

Visible Login $(\mathrm{Y} / \mathrm{N})$ ? Y

Logging Name...

Last caller was John Smith.

Local Sysop is NOT available for chatting.

Press [RETURN]:

Press [ctrl-s] to Pause, [spacebar] or [ctrl-c] to Abort

\section{Screen \#3}

On the LAM Bulletins:

5. 4-26-91 May 16 Membership Meeting

4. 4-26-91 May 1 Board Mtg.

3. 4-25-91 Science and Tech Foundation Support

2. 4-23-91 AKIA Office Hours

1. 4-14-91 New Uploads

$[R]$ ead, [P]ost, [E]dit, [K]ill, [Q] uit $(?=$ Help): Q 
End of mall

\section{Screen \#4}

[A] Command: Help

[ On the LAM BBS Command Sumnary

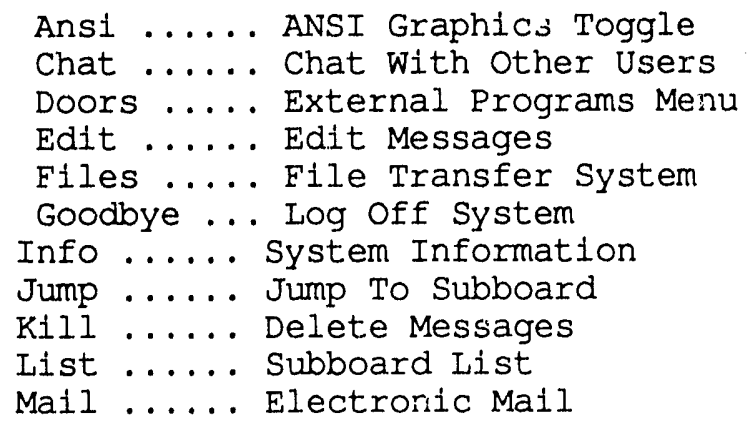

[H] Command: List

\section{Screen \#4}

Subboard Iist

\begin{tabular}{|c|c|c|}
\hline Board & es & Sub-Sysop \\
\hline A- General & 49] & SYSOP \\
\hline B- Alaska Science \& Tech Fdn & 3] & JOHN SIBERT \\
\hline 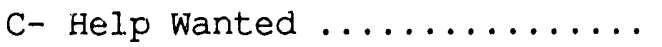 & 18] & DONNA ANDERSON \\
\hline D- Technical Help ........... & 10] & BRETT THOMAS \\
\hline E- Money - Where it is & 5] & ALLAN JOHNSTON \\
\hline F- Invention ideas ..... & 1] & DONNA ANDERSON \\
\hline G- Marketing 1deas, info & 2] & CHARLES LOCHNER \\
\hline Diary ........... & 58] & DOUG ASKERMAN \\
\hline
\end{tabular}

Enter Sub Letter [? for List]:

[H] Command: Goodbye

\section{Log Off System}

\section{Are You Sure $(\mathrm{Y} / \mathrm{N})$ ? Y}

Please leave us a quote:

$>$

Logoff at Mon, 4-29-91, 2:08 pm

Elapsed time $=1$ minutes

Thanks for calling On the LAM BBS.

(c) 1990 Searchlight Software, by Frank LaRosa 
Those familliar with the BBS community, and especially those who have used other BBS software, will appreciate the The LAM's long list of powerful functions and enhancements:

- Wordstar compatible full screen text editor makes it easy to enter and edit text and messages, even from a remote terminal.

- Unique user interface with built in Online Help facility gives both you and your users full access to the system's documentation.

- ANSI graphics support for flexible screen displays.

- Full security system with 255 levels of access.

- Up to 24 message subsections, up to 300 messages per section.

- Complete Electronic Mail system.

- SYSOP utilites allow you to adjust most aspects of the system to your liking and give you powerful mass deletion commands. You can control the entire system from a remote location.

- FILES system featuring alphabetical and date sorted directories, passwords and access levels, XMODEM and YMODEM transfers, archive directories, etc. FAST file transfers with advanced buffering techniques.

- DOORS system gives you infinite expandability: run almost any program from within your BBS, write your own commands (in any language), even give DOS commands from a remote terminal. The LAM is compatible with many of the popular PCBoard(TM) door programs. 


\section{The LAM BBS, CONTRASTED WITH OTHER BBS SYSTEMS:}

Before you begin using The LAM, we will provide a short explanation of some of the major design features of the BBS and how these compare to other BBS systems. It seems that all of the common BBS systems are based on a similar set of standards. The LAM was built to be readily accessible, user friendly and effective for "inventors". For that reason, the menu is patterned after many of the services that are "conventionally" provided in an inventors association office.

\section{Message System}

Many BBS's have a "flat" message system structure; that is, all the messages on the BBS are viewed as one long list, with the oldest messages at the beginning of the list, and the newest messages at the end. Still others have message sub-sections (often called Conferences, Subboards, etc.) that provide you with, essentially, several smaller lists of messages rather than one long list.

The LAM is, for the most part, similar to these systems. On Search light the message subsections are called "Subboards" and you can have as many as 24 of them, each with a different name and access level. In addition, you can assign a "Subsysop" to each message section who acts as the mediator for the discussion and has special privlidges to edit and delete messages under his/her domain. 


\section{Electronic Mail}

On most systems, sending a private message to another user is possible by specifying the name of the user to whom the message is directed. Such a message cannot be read by others, but it still appears on the message subsection with the other messages. Typically, you can find messages directed to you by performing an automatic search of all the messages in the system, looking for ones with your name on them.

The LAM uses a different approach. Rather than placing private messages in with the general message base, you send messages directly TO another person on the system. This operation is called Electronic Mail, and it is similar to the way real mail works. When you decide to send a personal message to someone, the system looks up their name and places your message into that user's personal "mailbox". To read a personal message, you inquire into your own mailbox to see what mail other people have sent to you. This method is a lot faster than the traditional approach and gives you an easy way to manage your own messages, separate from the main stream of things.

Incidentally, writing a public message on The LAM is known as "Posting" the message, and is done with a command called POST. Sending a private message is referred to as "Sending" the message, and is done through the MAIL command.

\section{Message Numbers}

Most BBS's assign a sequential message number to each message that is entered into the system. While this seems like a wholly obvious and logical way to organize a message base, anyone who's used a well established BBS has probably encountered the major deficiency in this kind 
of design. Even though old messages are deleted, message numbers continue in sequence, resulting in hard to remember four and five digit message numbers, even if there are less than 100 messages in the system. While most BBSs (as does The LAM) provide you with commands that let you access the most common operations without having to type numbers, large numbers are still unsightly and confusing, especially when, for example, there are only 10 messages between message 3000 and message 4000 .

We thought a better idea would be to have "floating" message numbers. On The LAM, the number of the highest message is always equal to the number of messages on the subboard. If, for example, a subboard contains 75 messages, the oldest message is message 1 and the most recent is message 75. What this really means is that messages don't actually HAVE numbersbecause when a message is deleted, all subsequent messages are "renumbered" one number down. So don't be suprised if what was message 50 one day becomes message 45 the next. That's the way the system works.

\section{Bulletins}

The LAM BBS has user-definable bulletins. Unlike other systems, where the bulletin feature is defined by the sysop, users can post bulletins. The bulletin function is actually a special message base with features that make it good for posting announcements and urgent messages. Complete details on how it works follow in the body of this manual.

\section{Quotes}

As with bulletins, quotes on The LAM is not a static file of quotes as found on some systems, but a function that stores and displays quotes left by the users of the system.

\section{Elow Control}


The term "flow control" refers to the flow of characters between the BBS and a remote terminal, and it's very important on an interactive system like The LAM BBS. During almost all operations. you can pause the output from the BBS by pressing control-s, and abort a message or listing by pressing the spacebar or control-c (to enter a "control" character, you hold down the key marked "Ctrl" on your keyboard and press the desired character, then release both keys. In this manual, control keys will generally be indicated with the $\wedge^{\wedge}$ symbol; in other words, $\wedge^{\wedge} \mathrm{C}$ means control-c).

Most BBSs have, or claim to have, this kind of flow control, but on The LAM we designed accurate flow control into every part of the system. Whereas some programs won't stop sending output until several seconds after you press ${ }^{\wedge} \mathrm{S}$, The LAM will almost always "stop on a dime". On many BBSs that we've seen, the ${ }^{\wedge} \mathrm{C}$ feature rarely works; on The LAM it works so well that it is sometimes the only way to exit from long listings and printouts. For example, after the screen fills up with text, most systems will stop and present a prompt such as "More? (Y/N/NS)" and take the respone. The LAM's "more" prompt simply says "more", and it takes any key as the response to continue sending output. There's no need to explicitly ask if you want more, because you can halt the BBS at any time by pressing ${ }^{\wedge} \mathrm{C}$ - and keep that thought in mind, because ${ }^{\wedge} \mathrm{C}$ (or spacebar) is a very powerful and oft overlooked feature of the BBS.

One final word about flow control: in practically every situation where the BBS requires you to answer an input prompt with a single character response, you need only type the desired character, NOT type the character and then press RETURN. You only need to press RETURN after typing a whole 
line of input. If you forget, and DO press the return key, it will be stored in memory (all input is fully "buffered") and used as the response to the NEXT input prompt. This can be quite undesirable and confusing, if you do not understand what's going on- so be a little careful at first.

There are many more interesting The LAM features, but rather than go into detail here, let's get started with the installation instructions.

These are the major points we want you to keep in mind as your system unfolds, and we'll get to the rest of the goodies in turn.

\section{Hardware Selected}

From: SYSOP Read: 22 times [2 Replies]

Subject: Computer purchase

Hardware specs for the new LAM computer. Will have invoice from Gateway 2000

Computer Co. at board meeting tomorrow night:

33 Mhz 80386

4 MB RAM

64K Cache RAM

1.2 MB 5.25" Drive

$1.44 \mathrm{MB} 3.5^{\prime \prime}$ Drive

$200 \mathrm{MB} 15 \mathrm{~ms}$ IDE Drive

16 Bit VGA w/ 1MB 14" VGA Monitor

2 Parallel, 4 serial ports

101 Key Keyboard

1-year on-site parts and labor warranty.

4 ea 2400 baud v.42bis modems from Zoom telephonics.

Software:

MS DOS 4.01, MS Windows 3.0, Desquiew, SLBBS 


\section{Appendix}

Developers/Designers Qualifications

Cooperative Agreements

Software Designs Special Features

For More Information - Additional Resources 


\title{
Developers/Designers Qualifications:
}

\author{
ROBERT L. CROSBY JR. \\ P.O. Box 77-2773 \\ Eagle River, AK 99577
}

\section{EDUCATION}

Houghton College, Houghton, NY; NYU, NYC 1961-1964 BSEE/MSEE Pgm.

USAF Technical School, Lowry AFB, CO.: Electronics. 1964-1965

\section{PROFESSIONAL AFFILIATIONS}

American Society of Heating, Refrigeration and Air Conditioning

Engineers, Inc. (ASHRAE)

Alaskan Academy of Engineering \& Sclences

Alaska Inventors Association, Inc. (member Board of Directors)

\section{PROFESSIONAL WORK EXPERIENCE}

Twenty years experience in A/E design, energy management or related field. Primary area of involvement since 1976 has been in mechanical systems design: HVAC, plumbing and utllities. Design projects include a wide range of residential, commercial and institutional projects. Have acted as project manager/ principal designer on over 200 projects since 1976, with majority of design work completed since 1984 produced using AutoCAD computer aided drafting/design (CADD) system.

Specific work experience includes project management, design andproduction of drawings and specifications, software development(CADD applications, design \& analysis tools), cost estimating,performance of economic analyses/energy studies, computer monitoring system development.

Project experience includes single- and multi-family housing, retall space, office buildings, maintenance \& repair facilities, schools (both urban and rural Alaska), churches, senior citizen centers, an 86-room hotel, restaurant/bar facilities, a fish hatchery, a solid waste transfer facility, a community freezer, a controlled environment growth chamber, a village waste separator.

\section{PROFESSIONAL HISTORY}

Self-employed in Anchorage since 1975 as a contract mechanical designer, with increasing emphasis on CADD related applications \& consulting. Major clients/associations (and related experience) during that time include: 
(Mar 1989- Present) - Design Lab, Inc, Architects, Planners: Project manager, mechanical design; CADD manager.

(Oct 1987 - Mar $1989 \quad$ USKH, Inc. Architects \& Engineers: Senior mechanical designer and CADD system co-manager (VAX based McDonnellDouglas software).

$(1986-1987)$

Livingston-Slone Inc., Architects \& Engineers: Responsible for AutoCAD system management,training and software development.

$(1983-1989)$

Alaskan Technical Consultants, Inc. (Merged USKH): Senior mechanical designer. Various commercial and instltutional projects, urban and rural Aiaska.

$(1981-1983)$

Adams, Morgenthaler \& Co: Senior mechanical designer, primarlly HVAC systems. Various commerclal and institutional projects, both urban and rural Alaska.

$(1978-1981)$

Tectonics, Inc: Project manager, mechanical design. Varlous commercial and institutional projects, both urban and rural Alaska.

$(1975-1978)$

Architectural drafting/design consultant, varlous A/E firms, private clients. Specialized in residential solar heating \& coollng design.

$(1972-1975)$

Employed as draftsman/designer with various $A / E$ firms, (Disciplines include architectural, clvil,structural, mechanical, electrical, electronics).

United States Air Force:

Electronics technician, Draftsman

BEGIN GROUP

RELATED PROJECTS

I have specific experience in the design of solar heating and cooling systems, energy recovery and storage systems, biologicalwaste water treatment systems, and have developed control systems for integrating alternative and conventional energy sources. Am especially interested in appropriate technology research and development, and have gained some recognition in this area. Specific projects of interest include the following:

1989 - Alaska Craftsman Home Program Demo Project, Golovin, Alaska: Design and implementation of remote computer monitoring system, and two year study to evaluate variety of innovative HVAC systems and cold-regions construction techniques for HUD Mutual Help Indian Housing. 
CLIENT: Bering Straits Regional Housing Authority

\section{7 -Winner of U.S. Dept of Energy National Award for Energy Innovation - (See Bloshelter description below.)}

1987 - Waste Separator for village of Kaktovik, Alaska: Under contract to ATC, designed system for mechanical separation of non-biodegradable sollds from organic wastes, prior to discharge into sewage lagoon.

\section{CLIENT: Alaska North Slope Borough}

1984 - Klukwan School, Klukwan, AK: As member of ATC staff, was responsible for design of unique HVAC system which uses no fan energy, but wind and thermal pressure differences only, to induce flow of ventilation air through building. Project was a winner of $1985 \mathrm{AlA}$ award.

\section{CLIENT: Minch, Ritter, Forest, Architects}

1982 - Winner of Home Design Competition sponsored by the Alaska Energy Center. Submitted was a design for an integrated bioshelter designed to provide a high degree of self-sufficiency at competitive cost. Features include:

* Blological water purification and nutrient recycliny systems integrating greenhouse and aquaculture systems. A balanced aquatic polyculture system is used for water reclamation.

* Aerobic composting tollet (original design).

* Multifuel heating system which uses the mass of thestructure and bioshelter circulatory system for heatstorage and distribution. Near $100 \%$ combustion efflclency is achleved by utilizing waste $\mathrm{CO} 2$ forenhanced plant growth.

* Induced draft ventllation system with heat recovery.

* Direct digital control and monitoring system.

(Construction was completed in July 1986, and we arecurrently living in the bloshelter. The project was selected by the U.S. Dept of Energy for a National Award for Energy Innovation in 1987.)

1981 - A prototype passive environmental freezer for the village of Holy Cross, Alaska. An electronic control system operates dampers to store the winier "cold" in brine tanks to keep food frozen throughout the summer months.

\section{CLIENT: Tanana Chiefs Conference, Fairbanks, AK}

1980 - Mechanical system for a controlled environment "growth chamber" at Anchor Point, Alaska. Temperature, humidity,carbon dioxide, and light levels are automaticallycontrolled. No auxiliary heating or cooling equipment was installed in the facility: instead, temperatures are maintained by storing waste heat from the HID lights inphase-change materials during the "on" 
cycle, to bereleased to the growing space during the "off" cycle.

CLIENT: Anchor Renewable Farms, Anchor Point, AKENDF

\section{Software Designs Special Features}

\section{BBS COMMAND SUMMARY}

\section{SUMMARY OF MAIN MENU COMMANDS}

The following pages contain a command-by-command summary of the major commands that are available at the BBS's main command prompt.

For on-line help with any command press the first letter of the command you need an explanation of, and type the "?" key.

To execute commands, press the first letter of the command, followed by the RETURN key.

\section{QUICK GUIDE TO COMMANDS}

The following chart shows which commands are associated with the various operations that the BBS performs:

Operation Command(s)

Access Subboards LIST, JUMP, NEW

Read, Post, \& Manage Public Messages READ, POST, EDIT, NEW, KILL

Send \& Read Mail MAIL

Use Bulletins

BULLETIN

Display \& Change Options OPTIONS, TIME

Show User Lists \& Info

Access Upload/Download System

Access External Programs

End Session

USER

\section{FILES}

GOODBYE

The LAM BBS's main menu looks like this:

[ On the LAM BBS Command Summary ]

Ansi ...... ANSI Graphics Toggle New ...... New Message Scan

Chat ...... Chat With Other Users Options ... Your Options/Stats

Doors ..... External Programs Menu Post ...... Post Public Message

Edit ...... Edit Messages Quotes .... Logoff Quotations

Files .... File Transfer System Read ...... Read Public Messages

Goodbye ... Log Off System Scan ...... Scan Message Titles

Info ...... System Information Time ...... Time Information

Jump ...... Jump To Subboard User ...... User Lists \& Info

Kill ...... Delere Messages Version ... System Version No.

List ...... Subboard List Who ....... List Current Users

Mail ...... Electronic Mail 1-Subop ... Subop Options Mode 
HELP

The HELP command prints a list of all the available commands, with a short description of what each command does. Notice that two commands, FILES and 2-SYSOP, contain their own submenus with additional commands; use the HELP command to get a list of the commands available at a particular menu.

The commands that begin with numbers rather than letters are special privlidged commands. These commands are only visible on the menu by users who can access them.

You can customize the HELP menu. See "SETTING UP TEXT FILES" in the Installation section of this manual.

\section{ANSI}

The ANSI command is used to select the desired Ansi graphics mode. Color, Monochrome, or no graphics can be selected. Select "None" when logging into the BBS from a remote terminal that is non-ANSI compatible; select Color or Monochrome mode respectively for Color or Monochrome ANSI terminals, or when logging in locally.

If ANSI is enabled, the BBS will use Ansi escape sequences to control output, allow eight-bit IBM graphics characters to be sent to the remote terminal, and enable you to use the full-screen text editor to enter mess ages.

You can use ANSI when logging in locally, even if you do not have the ANSI.SYS console driver installed (see your DOS manual). However, if you use text files that contain ANSI sequences, these will not be displayed correctly. We recommend installing ANSI.SYS at all times.

\section{BULLETIN}

This command displays the system's bulletins. Bulletins are a special message section that's displayed automatically each time a user logs on. Up to eight bulletins can be available at one time. Old bulletins, unless assigned a "protected" status by the SYSOP, are deleted as new bulletins are posted. Bulletins are always displayed in order from most to least recent, allowing you to view the latest messages quickly.

To read a bulletin, type a number from 1 to 8 . The [P]ost subcommand is used to post a bulletin (any validated user can Post). [K]ill is used to delete bulletins; as with messages, only the SYSOP or the person who posted the bulletin can delete it. Old bulletins are also deleted automatically when new ones are posted.

A special hidden command, [S]tatus, is available to SYSOPs. This command lets you "protect" and "unprotect" a bulletin; protected bulletins cannot be deleted.

The Bulletin facility is designed for important messages of general interest. Restricting the Bulletins system to 8 messages is a feature designed to emphasize the importance of the messages posted, and increase the likelihood that they will be read rather than passed over by the casual user. Both public messages and private mail also available (see the POST and MAIL commands); general text file libraries can be created with the FILES system, which provides a TYPE command for displaying text on the 
screen as well as several error-free download protocols.

\section{CHAT}

This command allows a remote user to page the system operator. If the SYSOP indicates that he/she is available (via the ALT-A command), paging occurs, otherwise the message "sysop not available" is displayed.

To answer a page at the local computer, press any key. To exit from the chat mode, press the ESC key.

\section{DOORS}

This command allows you to access features external to the BBS program that can be provided by adding new programs. To use DOORS, you must configure define the available programs via the the DOORS.DEF file; see the "DOORS SUBSYSTEM" section of this manual for details.

\section{EDIT}

The EDIT command can be used to edit an existing public message on the current subboard. Users can only edit message which they posted; subsysops can edit any message on their respective sub-boards, and sysops, of course, can edit any message in the system.

When editing, you can change the message's title, text, or both. In addition, the SYSOP can assign a "protected" status to a message. Protected messages are exempt from all "mass" message deletions (instances where more than one message is deleted at a time), but can still be deleted indi vidually.

Consult the "TEXT EDITING" section of this manual for further inform ation on using the line and full screen message editors.

\section{FILES}

This command executes the The LAM file transfer system, a subsystem that can be used to send and receive files to/from the bbs. The files system provides many subcommands and has its own HELP facility which can be used to get more information on the individual file system commands.

You must define the file directories with a FILEDIR.DEF file. See "FILES SUBSYSTEM" in this manual for further information.

\section{GOODBYE}

Use this command when you are ready to log off, or disconnect, from the BBS. The computer will prompt you to "Please leave us a quote". Type a short 1-line message and press RETURN (sysops may just press RETURN).

Note that is is considered good practice to always log off properly by using the Goodbye command, rather than simply hanging up. However, hanging up will not cause any damage to the system.

\section{INFO}

The INFO command displays a short message defined by the sysop.

\section{JUMP}


This command is used to access the various message subboards available on the bbs. Public messages are grouped by subject, and up to 24 seperate sub sections are available, indicated by the letters $[A]$ thru $[X]$ to the left of the command prompt. The Jump command will prompt you for the letter of the subboard you want.

To see the list of available subboards, either type "?" in response to the Jump command's prompt, or use the List command from the main menu.

KILL

The KILL command is used to delete public messages. Unless you are a sysop or subop, you can only Kill messages you have posted; otherwise you may delete any message. The system prompts you for the message number, prints the message heading, and confirms the command before proceeding with the deletion.

\section{LIST}

This command provides a list of the message subboards available on the systt I... Up to 24 subboards, indicated by the letters [A] thru [X] to the left of the command prompt, may be available at any time. The listing shows the title, number of messages, subsysop, and new message status of each board.

To access the messages on a particular subboard, enter the sub's letter in response to the prompt that follows the listing (you can also press the key while the list is still in progress). If you wish to jump to a subboard without viewing the list first, you may use the Jump command.

\section{MAIL}

The MAIL command is used to send private messages to other users, and to read the private messages that other users send to you. You can only send mail to someone who's name is registered in the BBS user file (ie. you can't send mail to "ALL", etc). You can only read mail that's been sent to you (unless you use the special SYSOP command to read user mail).

To use the mail facility, execute the Mail command. This will present you with a short menu of mail subcommands. The commands are-

[R]ead - this options lets you read your mail, if you have any. Each message in your private mailbox is displayed in sequence. After reading a message, a prompt appears with options to reply to the message, re-read it, forward it (send a copy of the message to another user or a public message subboard), and delete it. Please remember that all messages in your mailbox are private and addressed to you, therefore, mail should be deleted as soon as you are finished with it.

[S]end - allows you to send a message to another user. You must type the name of a user who is logged into the system, otherwise the command cannot proceed. You need not type the user's full name; you may type the first few characters, and the bbs will locate all users with matching names and prompt you for the correct one.

$[\mathrm{N}]$ ew - this command is exactly like [R]ead, except that only new 
messages are presented for reading.

[L]ist - lists the messages in your mailbox, showing the sender, date, and subject of each. You can choose a message from the list and start reading your mail from that message on.

[I]nquire - lets you examime the mail you've sent to another user. If your mail has not yet been received, you can edit it or delete it from the other user's mailbox.

Notes: Help in using the text editor is available from within the editor.

The Mail command is for private messages only. Refer to the Post command for information on sending public messages.

\section{NEW}

The NEW command gives you a listing of those subboards that contain new messages, ie. any messages posted after the most recent message that you've read on that sub. You are then prompted for the desired subboard letter; upon entering the sub, you will receive a scan of the new messages. You can automatically begin reading the messages by responding with a ' $Y$ ' to the "Read new messages now" query.

Refer to the Scan and List commands for further information.

\section{OPTIONS}

This command displays a variety of information about your account on the BBS. It lists the dates of your first and last logons, your access levels and time limits, the amount of uploading and downloading you have done, and the information you gave about yourself when you logged in.

As indicated on the Options screen, you may change your location, type of system, phone number, and password. If you are using an ANSI compatible terminal, you can press the up and down arrow keys to select a field (as indicated by the highlight bar), and edit it with the usual cursor keys (left, right, DEL, etc.), pressing F1 or $\langle$ ctrl-z> when done. Non-ANSI users can change fields by responding to the prompt with the proper field number. The "location" and "system type" fields have no actual bearing on the operation of the BBS; they are simply there to provide information about you to others who use the system.

Notice that, for security purposes, your password is not displayed on the screen except right after you enter or change it. If you alter your password, the system will confirm the new password before assigning it to your account.

\section{POST}

This is the command you use to enter a public message on the current message subboard. You are prompted to enter a 1 to 40 character title for the message, and then placed in the text editor to enter the text (documentation for the editor's commands is available from within the editor). Upon completion, your message is saved as the highest-numbered message on the subboard.

See also: Read, for reading back posted messages, and Mail, for sending private messages to other users. 


\section{QUOTES}

The quotes command displays, at random, a few of the one-line quotations left by users in response to the "Please leave us a quote" query that's presented as you log off the system.

\section{READ}

The READ command lets you read messages on the current subboard. You must enter the number of the first message you want to read. Notice that messages are always numbered sequentially; ie. if there are active 56 messages on a particular subboard, the first message is always number 1 and the last is number 56, regardless of the fact that message deletions may have taken place in the past.

After reading a message, you have several options. You may type ' $\mathrm{N}$ ' (or simply press RETURN) to read the next message. You can re-read the message by pressing ' $A$ '. You may reply to the message, either publically or privately; if the reply is public, it is appended to the current subboard, otherise the Mail facility is invoked to send the reply directly to the user who wrote the original message.

The message forwarding option can be invoked by pressing ' $\mathrm{F}$ '. This facility allows you to send a copy of the message you've just read to another place on the BBS: the destination can be a user's mailbox or a public message subboard. The forward command prompts you for the required parameters.

For more information, refer to: List and Jump for selecting other message subboards, Scan for scanning message titles, and New for reading new messages.

\section{SCAN}

Use the SCAN command to get a quick summary of the messages on the currently active subboard. The scan shows the message number, the date the message was posted, the name of the sender, and the title of the message. You may scan messages in forward or reverse direction by replying to the Scan prompt with ' $F$ ' for forward or ' $R$ ' for reverse. Note that you can abort a scan at anytime simply by pressing <spacebar $>$ or $<$ ctrl-c $>$ keys.

Refer to the Read command for reading messages, New for scanning new messages only, and List and Jump commands for selecting a new message subboard.

\section{TIME}

This command displays the current time and date, as well as the time which you logged onto the system, your elapsed time on the bbs, and the amount of time remaining in your current session.

\section{USER}

The USER command displays information about the registered users on the BBS. It has three subcommands:

[L]ist - gives you a list of the registered users, showing the users' 
names, dates of last login, locations, and system types (the type of computer or terminal they use). The list is alphabetical, and you don't have to read the list from the beginning to the end; you can start at any particular place by entering the first few letters of the name where you wish to start. Notice that you can abort the listing at any time by pressing the $<$ spacebar $>$ or $<$ ctrl-c $>$ keys.

[S]earch - allows you to search for a particular name in the user file. You need not type in the entire name; typing the first few characters is sufficient. If the name is found, it is listed along with information about the user.

[C]allog - this displays a list, in reverse order, of the names of the last 75 users to log into the system, including the times and dates of the calls. Again, you can abort the list early by pressing <spacebar $>$ or $<$ ctrl-c $>$.

\section{SUBOP (1)}

The SUBOP command, which can only be executed by sysops or sub-sysops, allows you to display and change the parameters for the current subboard. You use this commnand to initially setup your message subboards and to change or reconfigure them later. To execute this command, press the "1" key and then RETURN.

Subop displays four fields: Subboard title, subsysop, access level, and maximum message length. If using an ANSI terminal, you can change fields by moving the highlight bar to the desired field, and using the cursor keys to edit the field contents. In non-ANSI mode, type the number of the field you wish to change, and enter the new information.

Subboard title is any alphanumeric string defining the name of the subboard. The subsysop field must either be blank or the name of a valid user on the system. If nonblank, it defines the user who will have control over the contents and format of the subboard.

Access level defines the minimum access level that a user will need to access the subboard. An access level of 254 or 255 makes the board accessable only to sysops, and effectively "deactivates" the sub. This feature allows you to have private subs and create different classes of users. The first subboard (sub 'A') should always have an access level of zero, since ALL users will be able to access it by default upon logging in to the system.

Maximum message length defines the maximum length (in lines) of a message that can be posted. It normally defaults to 99 , but can be changed.

\section{READ SUBCOMMANDS SUMMARY}

\section{SUMMARY OF MESSAGE READ SUBCOMMANDS}

After reading a public or private message, the BBS prompts you with a number of options, to which you can respond by entering a 1-keystroke command. Here's a summary of the message disposition options:

\section{[A]gain}

The "Again" option is available with both mail and public messages. It clears the screen and re-displays the last message read. Use this option 
when you want to review a long message; it is also useful for redisplaying a message in case the message is garbled by line noise.

[N]ext

The "Next" subcommand is used to proceed to the next message in your mailbox or on the currently active subboard. If there is no next message, the READ command exits.

You may press the RETURN key instead of " $\mathrm{N}$ " to execute the next message subcommand.

[Q]uit

Select this option if you wish to quit reading messages and exit back to the main system prompt.

[R]eply

This option lets you reply to the message you've just read. If reading electronic mail, the reply is sent to the mailbox of the person who sent the message you've just read. If reading a public message, you have the option of sending the reply privately (as an electronic mail message) or publicly (as a posting on the current subboard). You are prompted with:

Is the Reply Private $(\mathrm{Y} / \mathrm{N})$ ?

Type "Y" if you wish to send a private message, " $\mathrm{N}$ " if you wish to post a public reply.

When you reply to electronic mail, and you don't delete the original message, the fact that you replied is recorded in the original message's header. The next time you read the message, it will display the number of times you've replied to that message as part of the header information. This helps you keep track of which messages have been replied to.

After writing the reply, you return to the read command in progress, and begin reading the next message in sequence.

[K]ill

This option appears only when reading public messages, and only if the person reading the message has permission to delete it. Selecting this option allows you to delete the message just read. It will ask you first if you really want to delete the message; type " $\mathrm{Y}$ " to kill it, "N" to keep it.

After deleting the message, the next message in sequence is displayed.

[F]orward

The "Forward" subcommand is used to forward a COPY of the message you just read to another place on the bulletin board. You may forward a message to a public message subboard or to a particular user's mailbox.

Pressing " $F$ " prompts you with:

\section{Forward to [U]ser or [S]ubboard?}

If you wish to forward the message to a public message board, press "S"; the BBS will then prompt you to enter the subboard letter of the board you wish to forward to. To forward the message to a user, type "U"; you will then be prompted to enter the name of the user to whom you wish to send the message. 
Forwarding a message to a subboard is the same as POSTing the message on that subboard. Forwarding to a user is the same as sending the message to that user via the MAIL command.

If the SYSOP or a cosysop is logged in, a third forwarding option appears in the menu of destinations:

Forward to [U]ser, [S]ubboard or [D]isk?

Selecting [D]isk allows you to write the message directly to a text file on your disk. It will prompt you to enter the filename, which may include a drive letter or directory path. If the file exists, the message is appended to the end of the file, otherwise a new file is created.

\section{THE DELETE PROMPT}

After reading each message in your electronic mailbox, and responding to it with one of the above subcommands, an additional prompt appears:

Delete this message $(\mathrm{Y} / \mathrm{N})$ ?

Press " $Y$ " if you wish to delete the message you just read; or "N" if you don't want to delete the message. Note that if you selected the [F]orward subcommand before deleting the message, the deletion will occur after the message is forwarded to its destination.

\section{EDITING}

\section{TEXT EDITING: INTRODUCTION}

The The LAM programs contain two text editors that are used whenever you enter a message or send electronic mail. The text editors, while not full-fledged word processors, contain many features and commands that allow you to easily compose, enter, and change short to medium length messages. In addition, you can use the BBS's text editing capabilites to edit text files on disk, even from a remote terminal.

A line-oriented text editor, similar in nature to the DOS EDLIN editor and to the editors found on many BBS systems, is provided for use with non-ANSI terminals. The line editor provides a fast and simple way of entering messages, with rudimentary editing facilities, and it works from almost any kind of remote terminal, from IBM PCs to older home computers to printing terminals.

The LAM also contains a full-screen text editor that resembles the types of editors most people are used to working with on the IBM PC. It gives you an exact representation on the screen of what your message will look like when read back, and it allows you to move the cursor anywhere on the screen and insert, change, or delete text with simple keystrokes. The commands used are a subset of the popular WordStar(TM) word processing program, so chances are you already know how to use The LAM's editor.

The main disadvantage of the full screen editor is that it's not compatible with all terminals- you must have an ANSI (or VT100) compatible terminal (or terminal emulator) to use it in remote mode. Fortunately, software to emulate ANSI terminals is available for most microcomputers- 
for the IBM PC, we recommend the excellent ProComm communications package (TM Datastorm Technologies Inc). In addition, you'll find that certain operations, such as PageUp and PageDown, are inherently slower when running from a remote terminal than when executed on a normal word processorthat's because the BBS can only send text at the speed of the modem being used, and cannot manipulate the screen at high speeds the way regular text editors can. However, The LAM makes extensive use of ANSI control sequences to minimize the amount of text that must be retransmitted to the remote screen. In local mode, it operates at speeds comprable to most full screen text editors.

The LAM determines which editor to use based on the status of the ANSI graphics mode. When ANSI (color or monochrome) is enabled, the full screen editor is used; otherwise, the line editor is used. You can change the status of the ANSI flag from the main menu with the ANSI command.

\section{USING THE LINE EDITOR}

When ANSI mode is not selected, the line oriented editor is used by the BBS. The editor is invoked whenever you POST a message or bulletin, EDIT an existing message, or send a message via the MAIL command.

When the line editor is running, you'll see the message

Enter text; type a period (.) when done. Max NNN lines.

followed by the number " 01 ". $\mathrm{NN}$ is the maximum number of lines that you may enter into the message (it can be anywhere from 5 to 250 , as defined on the subboard definition screen and in the CONFIG file).

You would now begin typing your message. As you type, you can correct errors by pressing the BACKSPACE key. There's no need to press the RETURN key when you reach the end of a line; instead, the editor automatically performs "word wrapping", that is, it breaks the line at the nearest word boundry and moves the last word on the line down to the next new line. As you type, you'll see the line numbers to the left of your text increase.

When you are done entering text, press the RETURN key once (so that the cursor is directly to the right of a line number). Then type a single period (.), and press RETURN again. This takes you out of the text insertion mode and places you at the line editor's subcommand prompt, which looks like this:

\section{Cont Edit Ins Del List Abort Save -?}

You can now enter an editor subcommand by typing the first letter of one of the above command words at the prompt. The commands work as follows:

Save

This is the command that saves the message you just entered (or edited) onto the disk. You use this command when you are done with the message, unless you've changed your mind and decided not to send the message at all.

Abort 
The abort subcommand can be used to throw away the message in case you decide you don't want to send it. It will prompt you to make sure that's what you really want to do, in order to insure that you don't abort the message by mistake.

Cont

The cont, or continue, subcommand lets you continue entering lines at the end of the message. It places you back into the text entry mode at the next highest line number.

List

This command can be used to list, or review, the message just entered. It will prompt you for a line number; if you enter 1, or simply press RETURN, the message is listed from line 1 to the end. If you wish the listing to start at a specific line number, you can enter that line number.

You can pause the listing with $\wedge^{\wedge} \mathrm{S}$, and cancel it with $\wedge^{\wedge} \mathrm{C}$.

Ins

This is the insert command. It lets you insert one or more new lines of text in between two existing lines. You are prompted to enter the number of the line that you want to insert BEFORE; for example, to insert between lines 11 and 12 , you'd give line 12 as the target of the insertion.

Once in insert mode, you can type as many new lines as you like. To exit back to the subcommand prompt, once again enter a period at the beginning of a new line.

Del

The delete command lets you delete one or more entire lines of text from the message. It prompts you to enter the starting and ending line numbers of the range of lines that you want to remove.

Edit

This command gives you a way to edit the individual characters on a line. It prompts you for the line number of the line you wish to edit, and gives you a choice:

\section{Change or Retype?}

Respond by pressing " $\mathrm{C}$ " to change the line, or " $\mathrm{R}$ " to retype it.

Retyping simply lets you re-enter the entire line of text from scratch. You can only retype one line at a time.

Change gives you the opportunity to edit portions of the line without having to re-key the entire line. It works by prompting you for a "source" and a "replacement" string. The "source" is a portion of the text, exactly as it appears in the line. The "replacement" is a string of text that will replace the source string. As an example, suppose we typed this into our message at line number 7:

07 Beethoven was bitten by a communist roach when he visited New York. 08 .

Obviously, we meant to type the word "salmon" instead of "roach". To correct it, we would edit the line and enter "roach" as the source string, and "salmon" as the replacement: 
Cont Edit Ins Del List Abort Save -? E

Line Number: 7

Change or Retype? C

Source String: roach

Replacement : salmon

Although the corrected line is not displayed on the screen, we could view it by using the "List" subcommand. The new version of the line would look like this:

07 Beethoven was bitten by a communist salmon when he visited New York.

Notice that you can replace a string with a string of a different length (or even a null string, in which case the original text is deleted entirely). However, the total length of the line cannot exceed 76 char acters.

\section{EDITING EXISTING MESSGAES}

The line editor can also be used to edit existing messages via the "Edit" command on the main menu. When used in this fashion, it operates identically to the above description, except that you'll go right to the "Cont Edit List..." prompt rather than to the "Enter Text" prompt. Saving the message results in the edited version of the message replacing the old version on disk. If you abort the edit, the changes you've made to the message, if any, are not saved; the original message is left intact.

\section{USING THE FULL SCREEN EDITOR}

When using an ANSI compatible remote terminal, or when logged in locally, you'll be able to enter and edit text via The LAM's full screen editor.

The screen editor signs on by displaying an inverse video bar near the top of the screen that contains this message:

\section{Editing, max NNN lines. Press $F 1$ or ${ }^{\wedge} Z$ to exit, $F 2$ or ${ }^{\wedge} \mathrm{J}$ for HELP.}

If entering a new message, the lower portion of the screen will be clear and the cursor will be positioned at the top of the text entry "window" on the screen. If editing and existing message, you'll see the first few lines of the message, with the cursor positioned at the first character in the first line. You are now ready to enter or edit text.

To enter text, simply begin typing. As with the line editor, you can correct single character mistakes as you type by pressing the BACKSPACE key. As you approach the end of a line, the text will "word wrap" down to the next line, so there's no need to press the RETURN key at the end of lines (unless, of course, you wish to type lines shorter than the width of the screen).

Unlike the line editor, which has subcommands for performing editing, you can edit text in the full screen mode interactively, using the cursor position keys and control keys. As you change the text, your screen is 
updated instantly to reflect exactly what the file looks like. If you've ever used a word processor on your IBM PC, you're probably very familliar with this method of editing: you'll need only learn a few of the commands and keystrokes that are specific to The LAM's editor.

\section{WORDSTAR COMPATIBILITY}

When we say The LAM's editor is "WordStar Compatible", what we mean is that, in most cases, we've used the same key sequences to perform the same editing tasks as the WordStar word processor. We picked WordStar because it's a well-known standard, and because its use of control key sequences (rather than menus or function keys) is particularly suitable for use with modems and remote terminals.

The LAM doesn't support ALL of WordStar's commands; in fact, it uses only a small subset of them. In addition, we've incorporated a few special commands and keystrokes that are unique to our editor.

\section{SCREEN EDITOR SUBCOMMANDS}

If you press either $\mathrm{F} 2$ or $\wedge \mathrm{J}$ (control-J) while in the editing mode, you'll see one of two help messages at the top of your screen. Press F2 again to see the second message. The help text looks like this:

\begin{tabular}{|c|c|}
\hline $\begin{array}{l}\text { Left } / \wedge S \text { - Cursor left } \\
\text { Right } / \wedge D \text { - Cursor right } \\
\text { Up } / \wedge E \text { - Cursor up } \\
\text { Down } / \wedge X \text { - Cursor down }\end{array}$ & 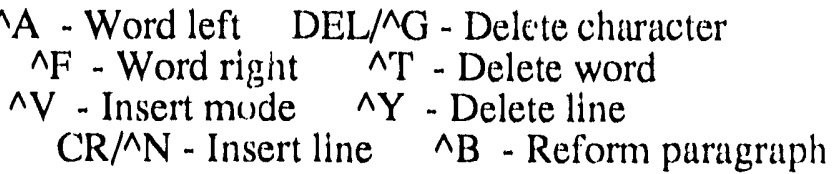 \\
\hline $\begin{array}{l}\mathrm{PgUp} / \wedge \mathrm{R} \text { - Page up } \\
\mathrm{PgDn} / \wedge \mathrm{C} \text { - Page down } \\
\text { Home/^QS - Begin of line } \\
\text { End } / \wedge \mathrm{QD} \text { - End of line }\end{array}$ & $\begin{array}{cc}\wedge \mathrm{QR} \text { - Top of file } & \wedge \mathrm{QY} \text { - Truncate line } \\
\wedge \mathrm{QC} \text { - End of file } & \wedge \mathrm{QL} \text { - Restore line } \\
\wedge \mathrm{L} \text { - Redraw line } & \wedge \mathrm{W} \text { - Redraw screen }\end{array}$ \\
\hline
\end{tabular}

These are the main editing commands. Many are self-explanitory, some need further study as we'll devulge below.

The $\wedge^{\wedge}$ character means CONTROL. To generate a control key, you hold down the key marked "Ctrl" on your keyboard, then press the indicated letter key, then release both keys.

Notice that some commands have two ways of being entered, either via a control key or via a function key. This is done because some remote terminals don't configure the function keys the same way they're supposed to be set up on the true ANSI terminal. If a key such as F1 or HOME doesn't work on your terminal, you can always use the control key instead.

Remember to press $\mathrm{F} 1$ (or $\wedge \mathrm{Z}$ ) to exit the full screen editor.

CURSOR MOVEMENT 
To edit text, you must first move the cursor to the character, word, or line that you want to change. The cursor movement keys accomplish this,

The four arrow keys (left, right, up, down) move the cursor one character in the indicated direction. If you move up or down past the top or the bottom of the screen, the screen will scroll to display the previous or next line of text. You cannot move the cursor past the beginning or end of the file, but you can append text to the end of the file.

Press the HOME key to move the cursor to the beginning of the current line. The END key takes the cursor to the end of the line.

The $\wedge \mathrm{A}$ and $\wedge \mathrm{F}$ keys move you left and right a word at a time. They don't move you off of the current line, however.

You can scroll a long message forward and backward one full screen at a time. Press the PgUp and PgDn keys for this. On a remote terminal, you must use ${ }^{\wedge} \mathrm{R}$ for pageup and ${ }^{\wedge} \mathrm{C}$ for pagedown.

Finally, you can move directly to the beginning or end of the text. Press ${ }^{\wedge} \mathrm{QR}$ to get to the top of the file, ${ }^{\wedge} \mathrm{QC}$ to go to the bottom.

\section{DELETION}

You can delete text by characters, words, or lines.

To delete the character under the cursor, press either DEL or ${ }^{\wedge} \mathrm{G}$. Pressing the BACKSPACE key deletes the character to the left of the cursor. When you delete text, the text to the right of the cursor moves left to fill the gap left by the deleted characters.

Text can be deleted a word at a time. Place the cursor on the first char acter in the word and press ${ }^{\wedge} \mathrm{T}$.

You can delete all the text on the current line from the cursor position to the end of the line. Press ${ }^{\wedge} Q Y$ to perform this function.

To delete an entire line, position the cursor anywhere in the line you wish to delete, and press $\wedge \mathrm{Y}$. When you delete a line, the remaining lines in the message move up to fill the gap left by the deleted line.

\section{INSERTION}

The The LAM editor supports either insert or ovestrike mode. In insert mode, text you enter in the middle of a line causes the text to the right of the cursor to shift, making room for the new characters. In overstrike mode, text overwrites any text underneath it, and no scrolling occurs. The editor is in insert mode each time it starts up, but you can toggle between modes with the $\wedge \mathrm{V}$ key.

When insert mode is active, an attempt to insert characters into a full line causes the line to be split in half at the cursor position. The message "Press $\wedge \mathrm{B}$ to reform paragraph" appears at the top of the screen. After typing your new text, press the ${ }^{\wedge} \mathrm{B}$ key and the subsequent text will 
move back up and reformat into a paragraph. In overstrike mode, press $\wedge \mathrm{N}$ to force a line break before entering new text.

You can also press the RETURN key to enter a new line in the text. In this case, the cursor moves down to next line, rather than remaining stationary as it does with $\wedge$ N.

\section{ERROR RECOVERY}

If you make a mistake, you can restore the contents of the current line provided the cursor has not left the line. Press $\wedge^{\wedge} \mathrm{QL}$ for this function.

\section{REFORMATTING TEXT}

After performing many insert and delete operations, your text will no doubt have lost its original format: you'll have some lines that are shorter than others. Never fear; you can reformat any continuous piece of text back into neat paragraph form by using the $\wedge \mathrm{B}$ command. Simply move the cursor up to the first line in the paragraph and press $\wedge \mathrm{B}$. The editor will format all the subsequent text into a paragraph, until it reaches a blank line or a line that begins with a space or another non-alphabetic character.

\section{REDRAWING THE SCREEN}

One of the pitfalls of using a full-screen editor via modem is that modems often generate spurious characters due to excessive noise on the phone lines. Even with good modems and clean lines, you're bound to experience modem garbage at least once in a while. If it happens while you're using the full screen editor, the results can be devistating: you'll see extra characters on the (remote) screen, your cursor will move, but the editor won't know about it, and will continue to operate under the assumption that your screen still looks the way it did before the "line hit" occured.

Fortunately, the The LAM editor provides ways to recover the screen display when this happens. If a modem error effects only the current line you're typing on, press ${ }^{\wedge} \mathrm{L}$. This command will resend the line to your terminal, and clear any garbage that might be present at then end of the line. If there are a lot of errors on several lines, you can redraw the entire screen. Simply press $\wedge W$. The editor will clear the remote screen, resend the text window, and position the cursor in its rightful location.

If you experience a lot of noise on a particular call, using the full screen editor can become extremely frustrating. In this extreme case, you can always fall back to the line editor; simply disable ANSI graphics, and enter the message again.

\section{ANSI COMPATIBILITY}

The LAM's full screen editor actually has three modes of operation, designed to allow it to interface with a variety of popular terminal software. When editing remotely using an ANSI terminal, certain operations (such as inserting and deleting lines) require that the terminal software implement the appropriate ANSI escape sequences that will cause the remote screen to scroll appropriately. Unfortunately, many terminal programs are 
geared for more traditional BBS systems without full screen editors, and thus do not support the required commands. To work with such terminals, The LAM includes a "Generic" ANSI mode. To select the editor's mode of operation, type the "Options" command (on the BBS main menu) and adjust option number 5, called "Ansi Compatibility Mode". There are three possible settings: Generic, ProComm, and Full.

In Generic mode, the The LAM editor minimizes the types of ANSI commands sent to the terminal to a small subset of the most widely supported ones. Instead of scrolling the screen and performing inserts and deletes directly on the terminal, The LAM redraws portions of the screen when a change needs to be made. Of course, the time it takes to continuously redraw the screen can range from long to unbearable, depending on the speed of the modem used; but all of the supported features will work correctly.

In Full mode, The LAM will edit with the full set of ANSI commands for the fastest possible output, and will require a full featured ANSI terminal. The remaining mode, ProComm, is similar to Full but takes into account several quirks found in the ProComm (TM Datastorm Technologie:s) terminal software.

The New User Registration procedure displays information about ANSI modes, and prompts each new user for the correct mode as they log in.

\section{ADDITIONAL THOUGHTS ON FULL-SCREEN EDITING}

Notice that, when you're using the BBS in ANSI mode, the above full screen editing commands are actually available everywhere in the system where a line of input is required. This includes entry of message subjects, user names, etc.; you can edit the line of text being typed with all of the commands that pertain to editing of the current line, including the ${ }^{\wedge} \mathrm{L}$ command to redraw the line.

\section{USING PREPARED TEXT FILES}

Sometimes, instead of typing a message by hand, you'll want to insert a previously written file of text into a message, bulletin, or electronic letter. There are several ways to accomplish this with the The LAM's editors.

\section{UPLOADING}

The simplest way to insert a text file into a message is to log on from a remote location using a terminal that's capable of sending lines of text from a file (this is sometimes referred to as "ASCII Upload" by the terminal's software). The editor doesn't know that you aren't really typing the message; it simply accepts the text coming from the terminal as if it were keyboard input, regardless of the fact that the terminal is really sending text from a file. The message editor is able to accept text at the fastest rate it can be sent by the terminal.

There are a couple of things to look out for when using this method of sending text. First, the text file should not contain lines longer than 76 characters; if it does, the BBS will try to wordwrap the text, resulting in 
a sloppy format. Also make sure the text doesn't exceed the maxium line length of the message.

Some communications programs won't show a correct display when you upload text to the full screen text editor. In particular, ProComm(TM) shows an excessive amount of control characters on the screen, even though the upload proceeds normally. The solution is to press $\wedge \mathrm{W}$ after the text has been sent.

You can't send text that contains embedded ANSI escape sequences, such as text that moves the cursor around on the screen or effects color changes (although eight-bit IBM graphics characters CAN be used). Since the full screen editor interprets these sequences as commands, not as part of the message, ii $v$ "l cause undesirable things to happen to the message. If you want embedded control codes, use one of the other methods for inserting text into messages.

\section{LOADING FILES DIRECTLY}

The text editors feature a special function, executable only by SYSOPs, that lets you load a text file directly into a message. To use it, press $F 1$ and then " $L$ " for the full screen editor, or press " $F$ " at the subcommand prompt of the line editor.

The editor will prompt you for a filename, and load the text of the specified file into the message. You can give a full pathname and drive letter if you want.

\section{FILES SUBSYSTEM}

\section{The LAM BBS FILE TRANSFER SYSTEM}

\section{Introduction}

One of the most valuable features of the The LAM BBS is its file transfer system. With file transfer, The LAM gives you the ability to transmit files of any type from one computer to another over the telephone, with the capability to check for errors. It lets you set up multiple directories where users can "upload" a file or choose from a selection of files to "download". You can have as many as 26 directories active at once, and a different set of directcries for each message subboard on your system, giving you thousands of possible directory setups. As the sysop, you can assign different access levels to control what users have access to what files.

The The LAM's file system has many useful features: alphabetical and chronological directory listings, wildcard directories, global directory scans, Xmodem, CRC Xmodem, and Ymodem file transfers, passworu protection, and private file transfers. Users can view the internal directory of an ARChive file while online. SYSOPs can delete files, move files between directories, and import filenames from existing files on disk quickly and easily. You can even edit file descriptions after they've been entered. Plus, the The LAM's file transfer programs are the fastest availahle, due to advanced two-way interrupt driven RS232 handling. 


\section{USING THE FILE TRANSFER SYSTEM}

To use the file transfer system, log in to the BBS and execute the FILES command from the main menu. You'll see a message indicating that the files system is active, and a command prompt similar to the BBS's main menu prompt. From here, you can execute the FILES system commands, as we'll describe in a moment.

\section{FILES SYSTEM COMMANDS}

Accessing the FILES system presents you with a new command prompt and a new set of commands. The new prompt works the same way as the BBS's main command prompt; it allows you to enter commands by typing the first letter of the command or by scrolling through the commands with the SPACEBAR and BACKSPACE keys, it will list available commands via the "Help" command, and it will display an explination of each command when the "?" key is pressed. The name and letter of the currently active file directory is displayed to the left of the prompt. To return to the BBS, you use the "Quit" command.

\section{PROTOCOLS}

You can select an upload/download protocol using the Xproto command. Xmodem, CRC Xmodem, and Ymodem protocols are currently available.

\section{UIPLOADING}

Use the "Upload" command to upload files to the BBS. "Uploading" means to send a file FROM the remote terminal TO the BBS. The remote terminal must support one of the file transfer protocols used by the BBS.

Before an upload begins, the program checks all directories to see if a file of the same name already exists on the BBS. If the file is a duplicate, the message

File already exists. Thanks anyway.

is displayed, and the upload aborts. If the file doesn't appear in any of the BBS directories but a file of the same name exists on the disk in the directory where the upload will be placed, the message

\section{Filename exists}

appears, and the file transfer is similarly aborted.

Don't attempt to use the Upload command when logged in to the system locally. Instead, you can use the Get command to load existing files into your BBS's list of files available for downloading.

\section{DOWNLOADING}

Files in the currently active directory can be downloaded, or sent FROM the BBS and TO the remote terminal, via the Download command. Don't use Download during a local login.

If a user's download/upload ration exceeds the maximum value as defined in the CONFIG program, no downloading is allowed until the user has uploaded enough to reduce his or her ratio to within the alluwed range. Users who are "non-validated" (those who's access level is no larger than 
the default new user's access level) are prevented from downloading.

The approximate time for the download is computed before the file transfer begins. If the time to download the file exceeds the amount of time left in the current user's session, the download is not allowed.

\section{DIRECTORY LISTINGS}

There are several types of directory listings that can be gotten using the Files ant New commands. Wildcard, alphabetical, and date-sorted listings are available.

To switch from one download directory to another, use the List command for a list of available directories, and Jump to move between them.

\section{FILE MAINTENANCE}

Sysops and co-sysops can delete files, move files from one directory to another, and change the description associated with a file. The commands to execute these functions are Kill, Move, and Edit. These operations can also be performed by the user who uploaded the file.

\section{PASSWORD PROTECTION}

Passwords can be assigned to files on an individual basis using the Passwd command. Once a file is assigned a password, it cannot be downloaded or deleted without giving the password. Even cosysops must know the password of a protected file to download it; only the SYSOP can access the file without using the password.

A typical use of the Passwd option would be to allow two users to exchange a file privately. The sender would upload the file and assign a password to it. He or she would then communicate the password to the intended receiver (by sending a private message), who would then use the password to download the file. If more than one user needs to access the file, the sender gives the password to each person who needs to be able to download it.

\section{FILES COMMAND SUMMARY}

\section{SUMMARY OF FILES SYSTEM COMMANDS}

Here's a printout of the File System's main menu of commands, followed by an explanation of what each command does.

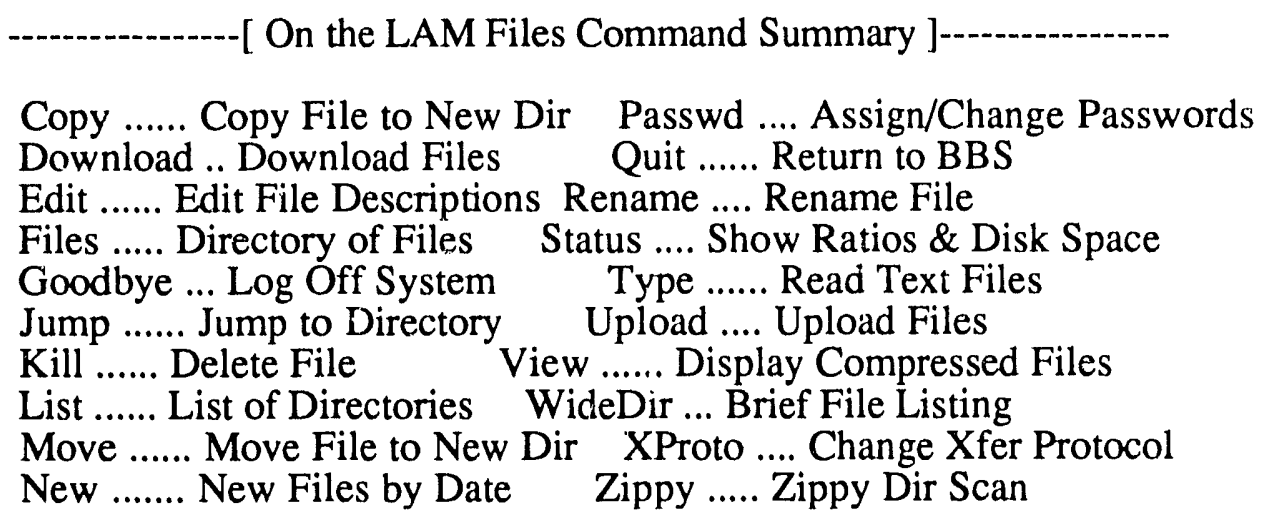



Show Add'l File Info

CHAT

This command allows a remote user to page the system operator. If the SYSOP indicates that he/she is available (via the ALT-A command), paging occurs, otherwise the message "sysop not available" is displayed.

To answer a page at the local computer, press any key. To exit from the chat mode, press the ESC key.

\section{DOWNLOAD}

The Download command is used to transfer files from the BBS to the remote terminal. The remote computer or terminal must be capable of receiving the file using one of three possible transfer protocols: Xmodem, CRC Xmodem, or Ymodem. If no protocol has been previously selected when you give the Download command, you will be prompted for a protocol; otherwise, the protocol used last is used.

To begin downloading, enter the name of the file you wish to receive. You must enter the full filename. ONLY FILES IN THE CURRENT DIRECTORY may be downloaded; if you wish to download a file from a directory other than the one that's current, you must Jump to that directory first. When the BBS displays the message "Ready to send", the file transfer is ready to begin. However, before you can receive the file, you must give your terminal the proper command. Usually, this is done by selecting an option to receive files on a menu.

A file may be password protected. If you attempt to download such a file, you will be prompted for a password, and the download will proceed only if you provide the correct password.

To get a directory of the files available for downloading, use the Files and New commands.

\section{EDIT}

This command allows you to change the 40-character description associated with a file. You can only change a file that you uploaded, unless you have sysop status, in which case you may edit any file's description.

\section{FILES}

The Files command displays a listing of files available for downloading. It prompts you to enter the "Starting Filename or Wildcard" of the directory you wish to view. There are three ways to respond to this prompt:

Pressing RETURN without typing anything gives you a list of all files in the current directory. The list is in alphabetical order and shows the filename, date uploaded, size in Kilobytes, and a description of the file.

Typing a filename, or the first few characters of a filename, WITHOUT a wildcard, lists files in alphabetical order starting with the filename entered. For example, if you enter " $L$ ", you will get a list beginning with the files that start with the letter $\mathrm{L}$, and continuing to the end.

Finally, you may type a wildcard (a filename containing one or more 
"*" or "?" characters). In this case, only those files that match the wildcard specification are shown. In addition, the prompt "Search all directories $(\mathrm{Y} / \mathrm{N})$ ?" appears. If " $\mathrm{Y}$ " is selected, the program will list all files in all directories that match the wildcard; otherwise, only files in the current directory are listed.

A " + " sign displayed in the file listing indicates a password protected file. You can only be download such a file if you know the password.

You may abort any file listing at any time by pressing the <spacebar> or <ctrl-c> keys. Also, refer to the "New" command for information about date-sorted file listings.

\section{JUMP}

This command is used to access the various file directories available on the system. Up to 26 directories are available, indicated by the letters $[A]$ thru $[Z]$ to the left of the command prompt. The Jump command will prompt you for the letter of the directory you want to access. You can only download files in the "current" directory.

To see the list of available directories, either type "?" in response to the Jump command's prompt, or use the List command.

\section{KILL}

The KILL command deletes a file. If you have Sysop or Co-Sysop access, you may delete any file; otherwise, you can only delete a file if you uploaded it.

\section{LIST}

The LIST command gives you a list of available directories. In order to view or download files in a specific directory, you must make it the current directory; you do this by responding to the List command's prompt with the directory letter, [A] thru [Z], or by using the Jump command (see Jump).

\section{MOVE}

Use the MOVE command to transfer, or move, a file from one directory to another. Actually, the file is copied into the destination directory and then the original file is deleted.

Users with Sysop access can move any file. Otherwise, you can only move your own files (files you have uploaded) into directories that you have access to.

\section{NEW}

The NEW command allows you to get a listing of "new" files since the last time you logged on, or since any specified date.

To use the New command, answer the prompt with the desired target date. The date shown in brackets, which is the date you last logged on, is used as the default date if you press RETURN. A second prompt appears, "Scan all directories $(\mathrm{Y} / \mathrm{N})$ "- if you enter $\mathrm{Y}$, all directories will be searched for new files, otherwise only the current directory is searched.

Dates are entered in the form month-day-year. You can use dashes, slashes, or any nonnumeric seperators. If you don't include the year (eg. 
"9/21"), it defaults to the current year.

The new file listing appears in reverse date-sorted order. It is often useful to run the NEW command with a very early target date, as this gives you a chronological file listing rather than an alphabetical listing as with the FILES command.

\section{OWNER}

This command prompts you for a filename and it displays the file's owner (the name of the person who uploaded the file) and the number of times that the file has been downloaded.

\section{PASSWD}

The PASSWD command lets you assign a password to a file. Once a password is assigned, only users who know the password are able to download the file.

You can only assign a password to a file that you have uploaded. If the file already has a password, you can only change it if you can give the old password. Note that the SYSOP can assign an automatic password to all newly uploaded files, thus preventing anyone from downloading a new file until it has been cleared by the sysop.

\section{QUIT}

The QUIT command returns you to the The LAM BBS Main Menu.

\section{TYPE}

The TYPE command allows you to read a text file. The output will pause every 24 lines and can be controlled with pause $(\wedge S)$ and break $\left({ }^{\wedge} \mathrm{C}\right)$ keys, just like when you read messages and other text.

You should only try to TYPE files that contain text. Typing other files will (usually) result in "garbage" characters being sent to your terminal. If this happens, press $\wedge^{\wedge}$ to abort the file.

\section{UPLOAD}

The Upload command is used to send files from a remote computer to the BBS. The remote computer or terminal must be capable of sending the file using Xmodem, CRC Xmodem, or Ymodem protocol. If no protocol has been previously selected when you give the Upload command, you will be prompted for a protocol; otherwise, the previously selected protocol is used.

To begin uploading, enter the filename of the file you wish to send. You must enter a legal MSDOS filename (8 character filename with optional 3 character extension). Do not include drive letters or directory pathnames. You cannot upload a file that already exists on the BBS (delete the file first).

If less than $200 \mathrm{~K}$ of disk space is available, the BBS will display the amount of space left and ask if you wish to continue. Press "N" if your file is larger than the amount of space available, or " $\mathrm{Y}$ " to go on.

When the BBS is ready to receive your file, it will display the message "ready to receive". What happens next depends on the terminal software that you use. In most cases, you must give a command to your software that tells it to load the file and begin sending it using the proper protocol. 
When the file transfer completes successfully, you will be prompted to enter a 1 to 40 character description of the file to be displayed so that others will know what information the file contains. When uploading a file as a SYSOP, you have the option of NOT giving the file a description. In this case, the file is not entered into the download directory, although it remains on the disk.

If the SYSOP has assigned a "default" password to the files system, your file will be password protected until it is cleared by the sysop.

The system "gives back" the amount of time it took you to upload the file by increasing your time limit by the time it took, so that you lose no time on the system for uploading.

\section{WIDEDIR}

This command gives you a "wide", four-across file listing which shows only the filename and file size in Kilobytes. It is similar to the DIR/W command in MSDOS. There is no parameter for WideDir; all files in the current directory are listed. You may abort the listing with ${ }^{\wedge} \mathrm{C}$.

\section{XPRO'TO}

This command lets you change the transfer protocol used for uploading and downloading files. The available protocols are Xmodem, CRC Xmodem, and Ymodem. If you attempt to upload or download a file without first selecting a protocol, the program will prompt you for one before continuing.

Once a protocol is selected, it is used by default for all subsequent file transfers until you change it with the XProto command.

\section{DOORS SUBSYSTEM}

\section{The LAM BBS DOORS SUBSYSTEM}

\section{Introduction}

The The LAM BBS DOORS system provides a method for linking external programs to your BBS. You can add additional features and capabilities to your system, such as games, user polls, extended sysop features, etc.

DOORS can be programs provided by The LAM Software, programs you write yourself, or programs writien by others. In addition, many general purpose MSDOS programs and utilities can be used as DOORS.

\section{MULTILEVEL DOORS MENUS}

As with the Files command, Doors lets you have a seperate menu for each of the 24 possible message subboards, if you desire.

create multilevel DOORS menus.

By multilevel, we mean that you can have a choice on the DOORS menu which, when selected, instead of running an external program, presents you with another menu of DOORS selections. You can divide your available Door programs into sections such as Games, Utilites, etc. As an example, you might have a DOORS menu that looks like this:

The LAM External Programs Menu 

1. The LAM Games Menu
2. The LAM User Utilites
3. The LAM SYSOP Utilites
4. DOS Command Shell

When a user presses " 1 ", another menu might appear that looks like this:

The LAM Games Menu

1. Online Adventure Games

2. Ron Chibnik's Slot Machine

3. PC-Hack

Some menu selections might run actual programs; others might bring up yet another menu with additional choices. 
For More Information - Additional Resources:

If you are interested in more information on the Alaska Inventors Association or the Linking Alaskan Minds project, please contact the

Doug Askerman, Executive Director Alaska Inventors Association

P.O. Box 201801

Anchorage, AK 995

(907) $272-7232$

LAM: (907) 694-6371

or

LAM Project Coordinator

Bob Crosby

Anchorage, AK 

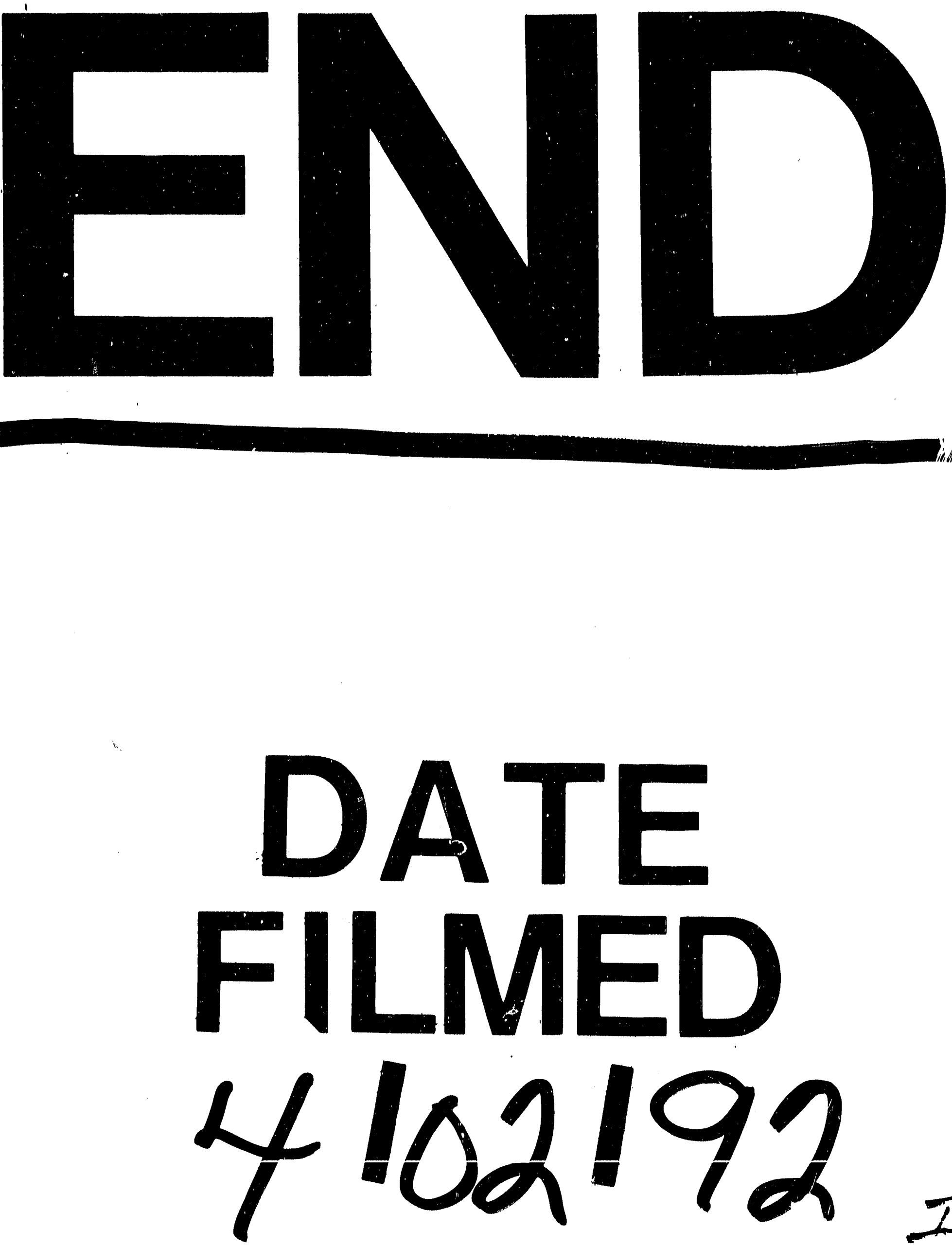

$I$. 
